EPJ Web of Conferences 31, 00003 (2012)

DOI: $10.1051 /$ epjconf/20123100003

(C) Owned by the authors, published by EDP Sciences - SIF, 2012

\title{
Liquid-gas phase transition in nuclear matter: Mean-field and beyond
}

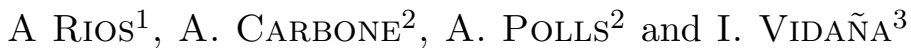

${ }^{1}$ Department of Physics, Faculty of Engineering and Physical Sciences, University of Surrey, Guildford, Surrey GU2 7XH, UK

${ }^{2}$ Departament d'Estructura i Constituents de la Matèria and Institut de

Ciències del Cosmos, Universitat de Barcelona, Avda. Diagonal 647, E-08028 Barcelona, Spain

${ }^{3}$ Centro de Física Computacional, Department of Physics, University of Coimbra, PT-3004-516 Coimbra, Portugal

\begin{abstract}
The liquid-gas phase transition in nuclear systems is a unique phenomenon, at the frontier of nuclear, many-body and statistical physics. We study this phase transition theoretically at the Hartree-Fock level and explore the available parameter space for the critical point by using several mean-field parametrizations. We find the latent heat of the transition and describe its temperature dependence from first principles. Using tools from many-body physics, we also describe the phase transition beyond the mean-field approximation and find a marked dependence on both the microscopic interaction and the many-body approximation scheme.
\end{abstract}

\section{Introduction}

Phase transitions are ubiquitous in science [1]. Nuclear physics is no exception, and a number of transitions of quite a different nature have been identified, from pion condensation [2] to quantum transitions associated to the nuclear shape [3]. Here we will focus on the theoretical description of

This is an Open Access article distributed under the terms of the Creative Commons Attribution License 2.0, which permits unrestricted use, distribution, and reproduction in any medium, provided the original work is properly cited. 
the liquid-gas phase transition, in which relatively dense (liquid) nuclear matter undergoes a drastic transition into a dilute (gaseous) system [4]. We will describe the phase transition within an ideal system (infinite homogeneous nuclear matter) in an attempt to constrain model dependences and potential uncertainties. This allows us to identify correlations between phase transition parameters which might be complementary to more empirical approaches [5]. Whereas there are clear limitations to this model, it has the advantage of allowing a clear-cut connection with mean-field parametrizations used in nuclear structure calculations [6] and with many-body approximations used to describe nuclear matter [7].

The search for the phase transition was originally motivated by experimental results in intermediate energy, heavy-ion reactions [8]. Several observables have been proposed as indicators of the phase transition [4]. In the near future, the new generation of radioactive beam facilities will lead to substantial advances, especially regarding the isospin dependence of the phase transition $[9,10]$. Theoretical approaches that can deal with finite temperature nuclear matter are therefore called for. A controlled and consistent approach in this direction is given by the self-consistent mean-field approximation, which is based on the same effective interactions used in nuclear structure calculations [11]. This approach can be alternatively casted as a finite temperature extension of density functional theory.

Before extensive finite nuclei calculations are implemented, however, the model dependence of the calculations needs to be properly assessed. This is already an issue at zero temperature and it remains so for hot nuclear systems. Among the possible sources of uncertainty, the dependence associated with the underlying effective interaction is capital, but has been scarcely investigated. Different effective interactions predict different thermodynamical properties and, thus, different liquid-gas critical points. In principle, a reliable determination of the critical point could lead to constrains on the properties of the effective interaction [5].

Homogeneous nuclear matter within a mean-field picture provides the simplest testing ground for such an analysis, since it lies within the thermodynamical limit and there is no need to account for finite size effects. Within the thermodynamical limit, however, one can go a step further and make a connection with the strong nucleon-nucleon (NN) interaction. To do so, however, a more sophisticated many-body description is needed, one that goes beyond the Hartree-Fock approximation. This is a more theoretically sound path towards linking thermodynamical and microscopic properties of nuclear matter, but it is also a more demanding one, due to the conceptual and numerical complexity of the many-body problem. We will outline here 


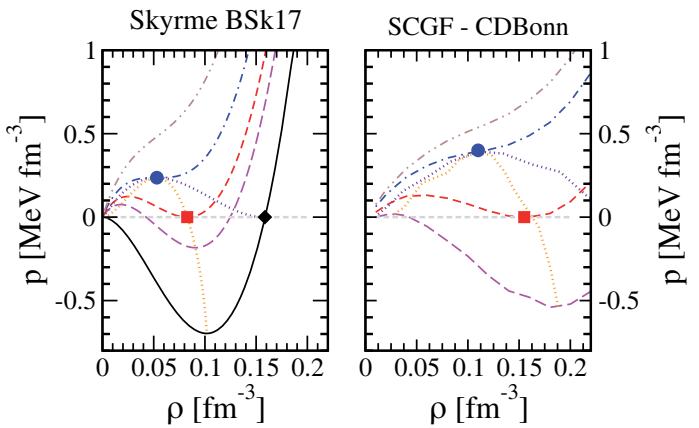

Figure 1: (Color online) Pressure isotherms of symmetric nuclear matter for different temperatures: $T=0$ (solid line), $T=10 \mathrm{MeV}$ (long-dashed line), $T_{f}$ (dashed line), $T_{c}$ (dash-dotted line) and $20 \mathrm{MeV}$ (dash-doubledotted line). The dotted lines represent the corresponding spinodal and coexistence regions.

our efforts to describe the liquid-gas phase transition with correlated (i.e. beyond mean-field) approaches. We will also provide a short summary on the status of the parameters of the phase transition as predicted by manybody approaches.

The liquid-gas transition in an homogeneous uniform system is only a first, crude approximation to the phenomena occurring in heavy-ion reactions and giving rise to multi-fragmentation. Clustering, finite-size effects and fluctuations dominate close to the critical point and none of these will be taken into account explicitly here. The picture that is provided by our calculations should thus not be taken as a meaningful description of the liquid-gas transition in nuclear systems, but rather as a simplified approach that provides a sound connection with more microscopic approaches. As such, this can be taken as a methodological contribution to test what information, if any, can be obtained from looking into the dependence of the phase transition properties on the microscopic properties of the system.

This contribution is divided in three separate sections. After this brief introduction, we will focus on a mean-field description of the phase transition, particularly looking at the dependence of the critical point on the underlying effective interaction. We will then describe the properties of the latent heat within this approach. Finally, we will summarise some of the results obtained with realistic many-body techniques, beyond the mean-field approximation.

\section{Phase transition at the mean-field level}

The density and temperature dependence of the thermodynamical properties of nuclear matter, obtained either within the Hartree-Fock approximation or within a more sophisticated approach, are non-trivial. These are obtained numerically and cannot be parametrized easily in an analytical form [6]. The 
numerical solution describes simultaneously two different phases: a gas at low densities and a liquid at densities close to saturation [12]. This behavior is easily visualized in the pressure isotherms of nuclear matter, as show in the two panels of Fig. 1. The left panel corresponds to results obtained with a typical Skyrme effective interaction (BSk17), while the right panel has been obtained with the Self-Consistent Green's Functions (SCGF) method based on a phase-shift equivalent $\mathrm{NN}$ interaction (CD-Bonn). In spite of the very different starting points of both approaches (effective interaction and meanfield approximation in one, microscopic NN force and many-body calculation in the other), the picture is qualitatively similar for both approaches.

Five characteristic temperatures have been chosen for illustrative purposes. At $T=0$ (solid lines, left panel), the pressure is a decreasing function of the density at low densities. This regime, for which $\frac{\partial p}{\partial \rho}<0$, corresponds to the spinodal region, where the system is mechanically unstable. Beyond the minimum, the pressure increases with density until it changes sign at the saturation point (diamond). Note that there is no $T=0$ line on the right panel, as SCGF can only be performed above the pairing regime [7]. As the temperature increases, the spinodal region, shown in dotted lines in Fig. 1, shrinks. The isotherm at which the pressure becomes a completely positive function defines the so-called flashing temperature, $T_{f}$. The flashing point (square) corresponds to the crossing of the spinodal with the zero pressure point, $p=\frac{\partial p}{\partial \rho}=0$. For an isolated system, without an external gas to stabilize it, this corresponds to the maximum temperature at which the system can still be self-bound.

For an infinite system, however, the system can exist above the flashing temperature because the gas and the liquid phase exert the same pressure on each other, according to the Maxwell criterion. Using this criterion, one can find the gas and liquid coexistence phases [1]. The coexistence region is shown as a dotted line in Fig. 1. Note that, at $T=0$, there is a coexistence between a zero-density gas and a liquid at saturation density. Increasing the temperature results into larger gas and smaller liquid densities. Eventually, the two densities meet at the critical point (circle), which occurs at the critical temperature, $T_{c}=15.5 \mathrm{MeV}\left(T_{c}=18.5 \mathrm{MeV}\right)$ for BSk17 (CDBonn). The critical isotherm is shown with a dashed-dotted line. Above the critical temperature, the system only exists in the gas phase and the pressure becomes a monotonically increasing function of density. This behavior is observed in the dashed-double-dotted line, which has been computed at $T=20 \mathrm{MeV}$ for both panels. Modern Skyrme and Gogny forces favor a critical temperature in the region $T_{c} \sim 14-17 \mathrm{MeV}$, and a flashing temperature within $T_{f} \sim 11-13 \mathrm{MeV}$ [6]. Microscopic many-body calculations 

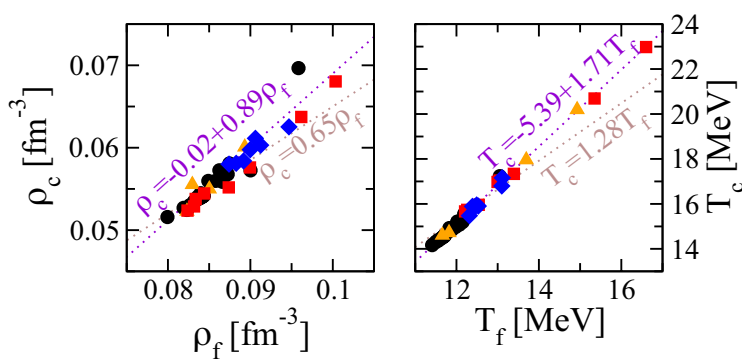

Figure 2: (Color online) Left panel: critical density versus flashing density for different effective interactions. Right panel: critical temperature versus flashing temperature for different effective interactions.

predict even larger windows, as we shall see in the following.

By computing the liquid-gas critical properties with a large set of different Skyrme force parametrizations, we hope to identify behaviors which are independent of the effective interaction. An example of such correlation is given in Fig. 2. A tight proportionality between the critical and the flashing densities (right panel) and temperatures (left panel) is found for a wide range of Skyrme and Gogny forces (blue circles). For Skyrme forces, we have differentiated between old forces with no effective mass (triangles), old forces with $m^{*} \neq m$ (squares) and new forces (circles). The flashing-critical correlation can be described either with a single linear parameter or with a linear regression with an offset, as shown in the Figure. Proportionality constants agree with those predicted by analytical models and the empirical relation $T_{c} / T_{f}=2 \rho_{c} / \rho_{f}$ is fulfilled.

The existence of such correlations is interesting at a purely theoretical level, as they unveil "universal" links between phase-transition quantities. From a more practical point of view, if experiments were able to determine with certainty a critical point, and if an extrapolation from finite to infinite systems were possible, one could determine the flashing point immediately from such correlations. Moreover, since a similar correlation exists between the critical temperature and a combination of parameters associated to both the equation of state and the effective mass at sub-saturation densities, one could also constrain the properties of the underlying meanfield parametrization $[5,6]$. Let us also mention that, in the context of a mean-field description of the phase transition, the critical exponents are those of the mean-field theory of critical phenomena, independently of the underlying mean-field [6]. 


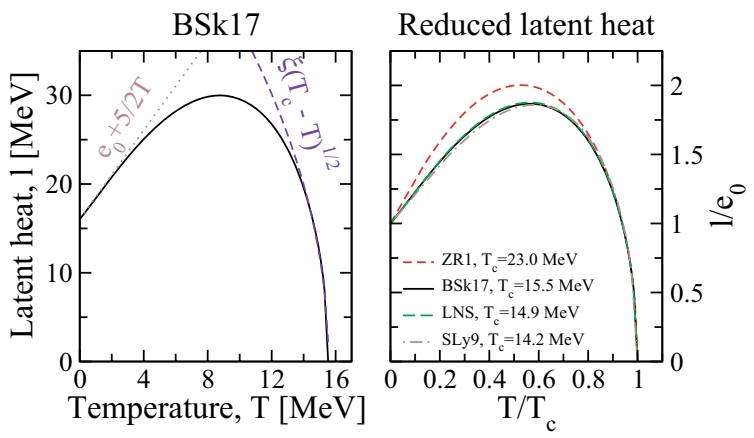

Figure 3: (Color online) Left panel: temperature dependence of the latent heat of symmetric nuclear matter for the BSk17 interaction. Right panel: reduced latent heat vs. reduced temperature for different Skyrme interactions.

\section{Latent heat of nuclear matter}

The latent heat provides a useful characterisation of the liquid-gas phase transition [13]. It is particularly appealing because it has been extracted from a variety of experiments, which suggest values of $l \sim 4-8 \mathrm{MeV}[5,8,14]$. In the case of infinite nuclear matter, the latent heat per particle has a simple definition: it accounts for the amount of energy needed to take a nucleon from the liquid to the gas phase. It can therefore be computed as a heat exchange between two phases that only involves the difference in entropies, $l=T\left(s_{g}-s_{l}\right)$, between the gas and the liquid phase at coexistence.

The latent heat, $l$, obtained in the mean-field approximation with the BSk17 Skyrme parametrization is reported in the left panel of Fig. 3 (solid line). The latent heat has a characteristic bump shape as a function of temperature. All other Skyrme parametrizations provide a similar qualitative behavior [13]. The latent heat matches the value of the binding energy at saturation, $e_{0}$, at $T=0$, and then rises for small temperatures. The initial rise is linear and the slope is actually independent of the Skyrme parametrization. As a matter of fact, the low-temperature behavior can be extracted from a careful analysis of the thermodynamics of the phase transition, yielding $l \sim e_{0}+5 / 2 T$. The analysis is valid for any homogeneous fermionic system undergoing a liquid-gas phase transition and provides a "universal" slope of the latent heat with a value of $5 / 2$ [13].

Further up in temperature, $l$ reaches a maximum and then drops to zero at the critical point, where the difference between the liquid and gas phases disappears. For BSk17, the latent heat maximum lies at $T=8.7 \mathrm{MeV}$ with $l_{\max }=29.9 \mathrm{MeV}$. Other Skyrme parametrizations also have a similarly large maximum of $l$. The existence of such a maximum is a consequence of the thermodynamics of the phase transition. The $T=0$ limit suggests that, to obtain interaction-independent results, one might normalise $l$ to 
$e_{0}$. A plot of the latent heat in reduced units, $l / e_{0}$, as a function of the reduced temperature, $T / T_{c}$, is presented in the right panel of Fig. 3 for a selected subset of four Skyrme forces. These represent a wide range of critical temperatures and maximum latent heats. In general, we observe that the dependence on the mean-field is eliminated to a large extent in the dimensionless plot. For all Skyrme forces, the latent heat tends to peak within a limited region of temperatures, $T / T_{c} \sim 0.5-0.6$, and the peak is narrowly distributed around the value $l_{\max } / e_{0} \sim 1.7-2$. When the temperature approaches the critical value, the properties of the system are characterised by critical exponents. In this regime, the latent heat is given by $\beta$, the same critical exponent that describes the order parameter which, in the Hartree-Fock approximation, is $\beta=1 / 2[13,15]$.

\section{Phase transition beyond the mean-field}

The mean-field approximation provides a simple picture of the thermodynamics of nuclear matter. Unfortunately, this approximation relies on phenomenological interactions (or density functionals) and the link with the underlying $\mathrm{NN}$ force is somewhat loose. A deeper understanding of the physics at play can be gained by looking into the thermodynamics of nuclear matter using, as a starting point, microscopic NN interactions. This cannot be done at the mean-field level, though, because of the strong short-range core of the interaction. Non-perturbative (beyond mean-field) techniques are needed to solve approximately the many-body problem. Here we will present results for two of these approximation schemes: the Brueckner-Hartree-Fock (BHF) and the SCGF approach.

To study the model dependence of the thermodynamical properties, we have performed calculations with two different NN forces (Argonne V18 and CDBonn) and two different approximation schemes (BHF and SCGF) in a large domain of densities and temperatures [7]. We then used the Maxwell criterion to find the properties of the liquid-gas phase transition. The numerics involved are much more demanding than those associated to mean-field calculations, which precludes us from performing some of the analysis of the previous sections (i.e. critical exponents are hard to find).

In Fig. 4, we summarise the properties of the phase transition for one NN interaction (CDBonn). The left panel shows the coexistence (circles) and spinodal (squares) regions of the phase transition for the BHF approximation. The critical temperature is around $23 \mathrm{MeV}$. On the right panel, the results for the SCGF approach predict a much lower critical tempera- 

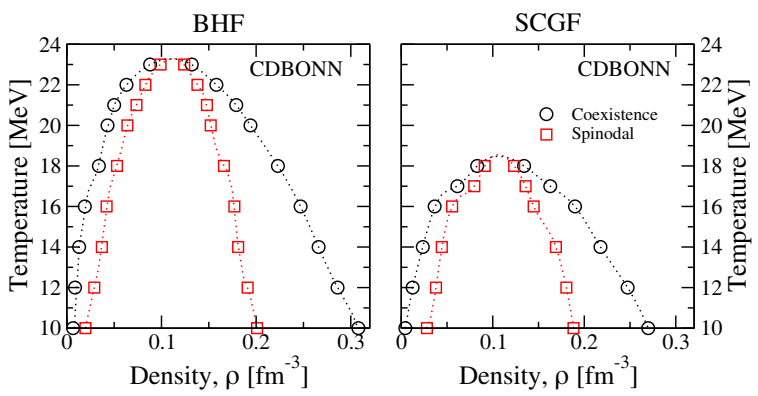

Figure 4: (Color online) Left panel: coexistence (circles) and spinodal (squares) regions for the CDBonn interaction within the BHF approximation. Right panel: the same within the SCGF approximation.

ture (around 18.5 MeV). This highlights the differences in the many-body treatment of both approximations. The equation of state for SCGF tends to be more repulsive than that of BHF due to the inclusions of hole-hole correlations, which in turns results into a lower $T_{c}$ [7].

A more extended analysis of these results is presented in Table 1 where we summarize the status of the phase transition parameters as obtained by us and by different groups over the last decade. This is by no means a complete list, as it only includes non-relativistic many-body calculations which use phase-shift equivalent potentials as a starting point. It provides, however, a good idea of some of the general trends.

We focus here on the critical temperature, density and the dimensionless ratio $\gamma_{c}=p_{c} / T_{c} \rho_{c}$, which characterises the critical point $\left(\gamma_{c}=0.375\right.$ for a classical van der Waals gas). Overall, we observe a rather wide variation in the range of allowed critical points, quite larger than the range observed for different mean-field parametrizations [7]. Microscopic approaches predict critical densities in the range $0.05-0.11 \mathrm{fm}^{-3}$, a variation of a factor of 2 . Similarly, all microscopic approaches coincide in providing a critical ratio which is well below the van der Waals value. Interestingly, these values are also below the minimum mean-field prediction $\left(\gamma_{c} \sim 0.28\right)$, which suggests that the transition is indeed somewhat different for mean-field and beyondmean-field approaches.

The critical temperature is perhaps the more characteristic property of the transition and microscopic approaches predict a rather large set of allowed values, from 11 to around $23 \mathrm{MeV}$. As mentioned earlier, the differences between the many-body approximations can cause this large variation. Similarly, different underlying two-body NN forces are associated to different critical points, even within the same approximation (see, for instance, the SCGF results in Table 1). It is also interesting to note that three-body forces $(3 \mathrm{BF})$ can substantially modify the phase transition [18]. Generally, the inclusion of $3 \mathrm{BF}$ s tends to lower the critical temperature: for BHF cal- 
Table 1: Critical point parameters for different many-body approximations and NN interactions.

\begin{tabular}{ccccc}
\hline \hline Approach & Potential & $\begin{array}{c}T_{c} \\
{[\mathrm{MeV}]}\end{array}$ & $\begin{array}{c}\rho_{c} \\
{[\mathrm{MeV}]}\end{array}$ & $\begin{array}{c}\frac{p_{c}}{T_{c} \rho_{c}} \\
{\left[\mathrm{fm}^{-3}\right]}\end{array}$ \\
\hline BHF [16] & Argonne V14 & 21 & $0.07-0.09$ & \\
BHF [16] & Argonne V14 + 3BF & 20 & $0.07-0.09$ & \\
BHF [17] & Argonne V18 & 16 & 0.08 & \\
BHF [17] & Argonne V18 + 3BF & 13 & 0.06 & \\
BHF [7] & Argonne V18 & 18.1 & 0.08 & 0.28 \\
BHF [7] & CDBonn & 23.3 & 0.11 & 0.28 \\
SCGF [7] & Argonne V18 & 11.6 & 0.05 & 0.14 \\
SCGF [7] & CDBonn & 18.5 & 0.11 & 0.20 \\
SCGF [18] & CDBonn & 18 & 0.11 & 0.22 \\
SCGF [18] & CDBonn + 3BF & 12.5 & 0.10 & 0.12 \\
SCGF [18] & Nijmegen & 20.5 & 0.10 & 0.26 \\
SCGF [18] & Nijmegen + 3BF & 11.5 & 0.09 & 0.14 \\
Variational [19] & Argonne V18 + 3BF & 21 & 0.05 & \\
\hline \hline
\end{tabular}

culations the effects is small, but for SCGF the reduction can be as large as $9 \mathrm{MeV}$. Similarly, the critical density is reduced by around $0.01 \mathrm{fm}^{-3}$, whereas $\gamma_{c}$ decreases by about 0.1 when $3 \mathrm{BF}$ are taken into account in SCGF calculations.

Overall, such a wide range of critical parameters is a reflection of the differences in many-body approximations and underlying $\mathrm{NN}$ interactions. These are manifestly shown in finite temperature nuclear physics due to the interplay of density and temperature effects in the liquid-gas phase transition regime. Improvements upon such results need of long-range theoretical efforts as well as the interplay with experiments and, potentially, astronomical observation of hot degenerate matter in neutron stars.

\section{Acknowledgments}

This work has been supported by a Marie Curie Intra European Fellowship within the $7^{\text {th }}$ Framework programme, an STFC Advanced Fellowship, by grant No. FIS2008-01661 (MEC, Spain) and grant No. 2009SGR1289 from Generalitat de Catalunya. 


\section{References}

[1] Huang K 1987 Statistical Mechanics 2nd ed (John Wiley and Sons)

[2] Migdal A B 1973 Phys. Lett. B 45448

[3] Cejnar P, Jolie J and Casten R F 2010 Rev. Mod. Phys. 822155

[4] Borderie B and Rivet M F 2008 Prog. Part. Nucl. Phys. 61551

[5] Natowitz J B et al. 2002 Phys. Rev. Lett. 89212701

[6] Rios A 2010 Nucl. Phys. A 84558

[7] Rios A, Polls A, Ramos A and Müther H 2008 Phys. Rev. C 78044314

[8] Pochodzalla J 1997 Prog. Part. Nucl. Phys. 39443

[9] Tsang M B et al. 2001 Phys. Rev. Lett. 865023

[10] Sfienti C et al. (ALADIN2000 Collaboration) 2009 Phys. Rev. Lett. 102 152701

[11] Vautherin D 1996 Advances in Nuclear Physics, Vol. 22 (Springer US) chap Many-body methods at finite temperature, p 123

[12] Bonche P, Levit S and Vautherin D 1985 Nucl. Phys. A 436265

[13] Carbone A, Polls A, Rios A and Vidaña I 2011 Phys. Rev. C 83024308

[14] Bonnet E et al. 2009 Phys. Rev. Lett. 103072701

[15] Landau L D and Lifshitz E M 1980 Statistical Mechanics, Part 1 3rd ed (Butterworth-Heineman)

[16] Baldo M and Ferreira S 1999 Phys. Rev. C 59682

[17] Zuo W, Li Z H, Li A and Lombardo U 2004 Nucl. Phys. A 74534

[18] Somà V and Bożek P 2009 Phys. Rev. C 80025803

[19] Mukherjee A 2009 Phys. Rev. C 79045811 